\title{
Herschel/SPIRE colors of galaxies at $\mathrm{z}>2.5$
}

\author{
F.-T. Yuan ${ }^{1}$, V. Buat ${ }^{2}$, D. Burgarella ${ }^{2}$, L. Ciesla ${ }^{3,4}$, S. Heinis ${ }^{2,5}$, \\ S. Shen ${ }^{1}$, Z.-Y. Shao ${ }^{1}$ and J. -L. Hou ${ }^{1}$
}

${ }^{1}$ Shanghai Astronomical Observatory, CAS, 80 Nandan Road, Shanghai 200030, China email: yuanft@shao.ac.cn

${ }^{2}$ Aix Marseille Université, CNRS, LAM (Laboratoire d'Astrophysique de Marseille) UMR 7326, 13388, Marseille, France, ${ }^{3}$ University of Crete, Department of Physics, Heraklion 71003,

Greece, ${ }^{4}$ Institute for Astronomy, Astrophysics, Space Applications and Remote Sensing,

National Observatory of Athens, GR-15236 Penteli, Greece, ${ }^{5}$ Department of Astronomy, University of Maryland, College Park, MD20742-2421, USA

\begin{abstract}
We compiled a sample of 57 galaxies with spectroscopically confirmed redshifts and SPIRE detections in all three bands at $z=2.5-6.4$, and compared their SPIRE colors with SED templates from local and high-z libraries. We find that local calibrations are inconsistent with high-z observations. For high-z libraries, the templates with an evolution from $z=0$ to 3 can describe the average colors of the observations at high redshift well. Based on the templates, we defined color cuts to divide the SPIRE color-color diagram into different regions with different mean redshifts. We tested this method and two other color cut methods using a larger sample (783 galaxies) with photometric redshifts. We find that these color cuts can separate the sample into subsamples with different mean redshifts, but the dispersion of redshifts in each subsample is considerably large. Additional information is needed for better sampling.
\end{abstract}

Keywords. Galaxy evolution, high-redshift, Infrared galaxies

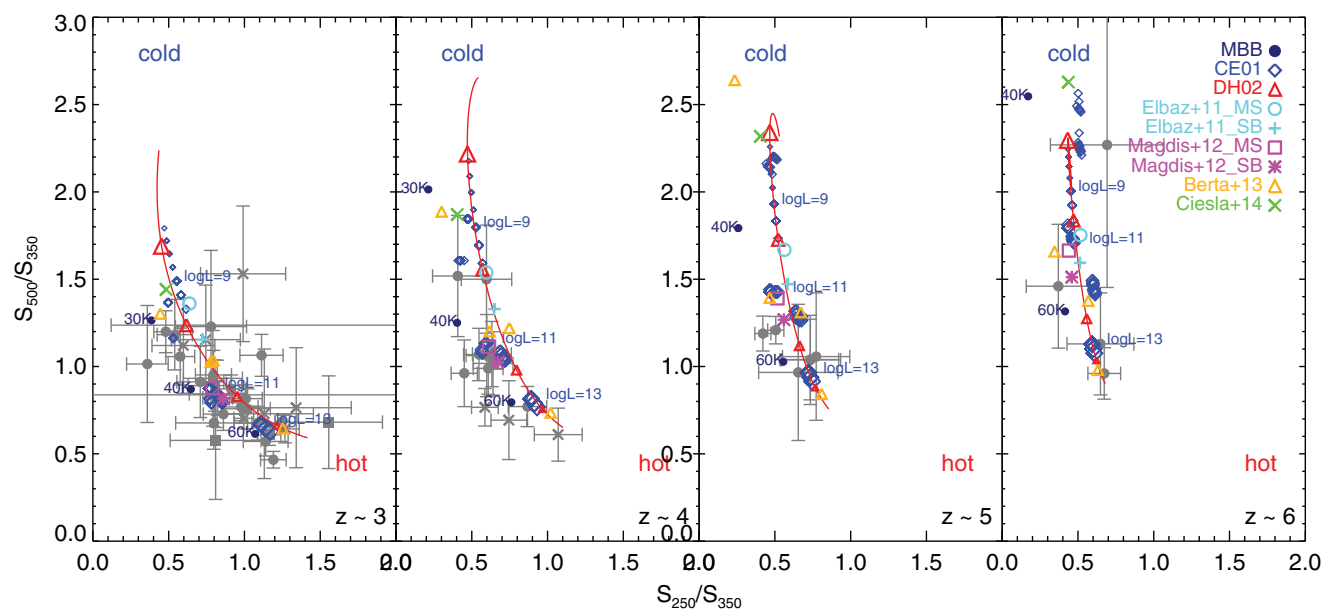

Figure 1. Comparison of the high-z observations and the SED templates on the SPIRE colorcolor diagram in different redshift bins $(z \sim 3,4,5$ and 6$)$. The observation data are in gray and the results of the templates are in color. Local templates have colder colors than the observed colors at high-z.

\section{Reference}

Yuan, F.-T., Buat, V., Burgarella, D., et al. 2015, A\&\&A, 582, A90 